Advanced Studies in Pure Mathematics 24, 1996

Progress in Algebraic Combinatorics

pp. 137-163

\title{
Covers of Complete Graphs
}

\author{
Chris D. Godsil
}

\begin{abstract}
.
We study antipodal distance-regular covers of complete graphs. The first part of the paper gives an introduction to the basic theory and the main constructions. In the second part, we turn to linear covers, which can be described geometrically.
\end{abstract}

\section{$\S 1$. Covers}

If we view a graph as a 1-dimensional simplicial complex then a cover of a graph is a cover in the topologist's sense. Fortunately there is a simple combinatorial approach.

Let $X$ be a graph with vertex set $V(X)$. An arc of $X$ is an ordered pair of adjacent vertices, and an arc function of index $r$ is any function $f$ from the arc set of $X$ into $\operatorname{Sym}(r)$, the symmetric group of degree $r$, such that

$$
f(a, b) f(b, a)=1
$$

for any $\operatorname{arc}(a, b)$ of $X$. Given an arc function $f$ of index $r$ on $X$ we construct a covering graph $X^{f}$ as follows. The vertex set of $X^{f}$ is the Cartesian product $V(X) \times\{1, \ldots, r\}$ and the vertices $(a, i)$ and $(b, j)$ are adjacent if

$$
i f(a, b)=j \text {. }
$$

Our condition on arc functions guarantees that $X^{f}$ is an undirected graph.

If $f$ is the identity on each $\operatorname{arc}$ of $X$ then $X^{f}$ is just $r$ disjoint copies of $X$. If $r=2$ and $f$ is equal to the non-identity element of $\operatorname{Sym}(2)$ on each arc then $X^{f}$ can be seen to be the direct product $X \times K_{2}$. The mapping $p$ from $V\left(X^{f}\right)$ to $V(X)$ such that $p(a, i)=a$ for all $i$ in $\{1, \ldots, r\}$ is called

Received March 28, 1995.

Support from grant OGP0009439 of the National Sciences and Engineering Council of Canada is gratefully acknowledged. 
a covering map. It can be shown to be a local isomorphism from $X^{f}$ to $X$, although this will play no role in our work here. The set of vertices $\{(a, i): i=1, \ldots, r\}$ is called a fibre of the cover. The subgraph of $X^{f}$ induced by a fibre is an independent set, and the subgraph induced by two fibres is either an $r$-matching or an independent set, according as $p$ maps the two fibres to adjacent or non-adjacent vertices in $X$.

If $f$ and $g$ are arc functions with index $r$ and $s$ respectively, we can define their product as follows. The ordered pair $(f, g)$ can be viewed as an arc function taking values in $\operatorname{Sym}(\mathrm{r}) \times \operatorname{Sym}(\mathrm{s})$, viewed as a permutation group on $r s$ points. Hence $\operatorname{Sym}(\mathrm{r}) \times \operatorname{Sym}(\mathrm{s})$ is a subgroup of $\operatorname{Sym}(r s)$, in its natural action, and therefore $(f, g)$ is an arc function of index rs. (The corresponding cover might not be connected, even if $X^{f}$ and $X^{g}$ are.)

The subgroup of $\operatorname{Aut}\left(X^{f}\right)$ consisting of the automorphisms that fix each fibre is called the covering group. It is an easy exercise to show that, if $X^{f}$ is connected, this group acts semi-regularly. We call a cover regular if the covering group acts regularly on the vertices in a fibre. It is another easy exercise to show that a 2-fold cover is always regular; its covering group is cyclic with index two. The size of a fibre is equal to the index of $f$, and is called the index of the cover. A cover of index $r$ is also called an $r$-fold cover.

If $X$ is connected and $T$ is a spanning tree in $X$ then it can be shown that any cover of $X$ is isomorphic to a cover $X^{f}$, where $f$ is the identity map on each arc of $T$. Arc functions of this form will be said to be normalised with respect to $T$. If $f$ is normalised with respect to some spanning tree of $X$ we define $C(f)$ to be the subgroup of $\operatorname{Sym}(r)$ generated by the elements in the image of $f$. We call it the connection group of $f$. The important properties of the connection group are summarised in the following combination of results from Section 7 of $[18]$.

Lemma 1.1. Let $f$ be a normalised arc function with index $r$ on a connected graph $X$ and let $C$ be the connection group of $f$. Then $X^{f}$ is connected if and only if $C$ is transitive. The covering group of $X^{f}$ is isomorphic to the centraliser of $C$ in $\operatorname{Sym}(r)$.

A graph with diameter $d$ is antipodal if the relation "is equal or at distance $d$ from" is an equivalence relation on its vertices. An antipodal cover is a cover such that the fibres are the antipodal classes; a covering graph may be antipodal without being an antipodal cover. Two vertices in distinct fibres of an antipodal cover of diameter $d$ must be at distance at most $d-1$. By way of example, if $f$ is an arc function of index two on $K_{4}$ and $f$ is not equal to the identity on any arc, then $X^{f}$ is the cube, 
which is an antipodal cover of $K_{4}$. The line graph of the Petersen graph can also be seen to be an antipodal cover of $K_{5}$ with index three.

Antipodal covers arise naturally in the study of distance regular graphs. If $X$ is a graph with vertex set $V$, let $X_{i}$ denote the graph with vertex set $V$, where two vertices are adjacent in $X_{i}$ if and only if they are at distance $i$ in $X$. Suppose that $X$ is distance regular with diameter $d$. Then $X$ is primitive if the graphs $X_{1}, \ldots, X_{d}$ are all connected, and imprimitive otherwise. If $X$ is imprimitive then either $X_{2}$ is not connected, and $X$ is bipartite, or $X_{d}$ is not connected and $X$ is antipodal. Further, it can be shown that there is a distance regular graph $Y$ such that $X$ is a covering graph of $Y$. If $Y$ has diameter $d$ then the diameter of $X$ is $2 d$ or $2 d+1$. The fibres of the cover are the antipodal classes of $X$, that is, the vertex sets of the components of $X_{d}$. The cube and the line graph of the Petersen graph provide two examples.

\section{$\S 2 . \quad$ Parameters of covers}

We now come to the main topic of this paper-antipodal distance regular covers of complete graphs. We will usually refer to these simply as covers. In this section we describe some of their properties. Any un-attributed results are taken from [18].

An antipodal graph with diameter two is complete multipartite, and consequently cannot be a covering graph unless each antipodal class is a single vertex. Thus it cannot be a non-trivial covering graph. Therefore an antipodal distance regular cover of $K_{n}$ must have diameter three. In addition to its index, such a cover has two further important parameters. If $X$ is distance regular then there are constants $a_{1}$ and $c_{2}$ such that any two adjacent vertices in $X$ have exactly $a_{1}$ common neighbours, and two vertices at distance two have $c_{2}$ common neighbours. Any covering graph has the same valency as the graph it covers, hence a cover of $K_{n}$ has valency $n-1$.

For antipodal distance regular covers of $K_{n}$ and index $r$ we have

$$
n-2-a_{1}=(r-1) c_{2} .
$$

To prove this, let $u$ be a fixed vertex in a cover of $K_{n}$ and let $v$ be adjacent to $u$. Then there are $a_{1}$ edges from $v$ to a neighbour of $u$ and hence there are $n-1-\left(a_{1}+1\right)$ edges joining $v$ to a vertex at distance two from $u$. On the other hand there are exactly $r-1$ vertices at distance three from $u$, each of these is at distance two from $v$ and so has $c_{2}$ neighbours in common with $v$. This yields the identity. We will refer to the triple $\left(n, r, c_{2}\right)$ as the parameter set of the cover. 
If $A$ is the adjacency matrix of a $\left(n, r, c_{2}\right)$ cover then the eigenvalues of $A$ are $n-1,-1$ and the two zeros $\theta$ and $\tau$ of the quadratic

$$
x^{2}-\left(a_{1}-c_{2}\right) x-(n-1)=0 .
$$

Because $\theta \tau=1-n$ it follows that, when $n \geq 2$, one of these zeros is positive and the other negative. When discussing covers of $K_{n}$, we will assume always that $n \geq 3$, and let $\theta$ be the positive zero of (2.2). The eigenvalues $n-1$ and -1 have multiplicities 1 and $n-1$ respectively. If $m_{\theta}$ and $m_{\tau}$ denote the multiplicities of $\theta$ and $\tau$ respectively then we have

$$
m_{\theta}=\frac{n(r-1) \tau}{\tau-\theta}, \quad m_{\tau}=\frac{n(r-1) \theta}{\theta-\tau}
$$

The fact that these multiplicities are integers is a strong constraint on the possible parameter sets $\left(n, r, c_{2}\right)$. The eigenvalues $\theta$ and $\tau$ are integers if $m_{\theta} \neq m_{\tau}$; if $m_{\theta}=m_{\tau}$ then we must have $\theta=-\tau=\sqrt{n-1}$.

There are three basic conditions on the parameters of a cover with parameter set $\left(n, r, c_{2}\right)$ :

(F1) $1 \leq(r-1) c_{2} \leq n-2$,

(F2) if $n$ is even, $c_{2}$ is even,

(F3) $m_{\theta}$ and $m_{\tau}$ are integers.

Here (F1) is an easy consequence of (2.1). For (F2), see Section 3 in [18]. The theory of association schemes provides the Krein conditions, which in this setting reduce to the inequality

$$
\left((r-1)^{2}-1\right)\left((n-1)^{2}+\tau^{3}\right) \geq 0 .
$$

If $r>2$, this reduces to

$$
\theta^{3} \geq n-1
$$

There are a number of other conditions that a parameter set must satisfy. Most of these are given in [18], see also [16], [17].

It is natural to partition the parameter sets of covers by the value of $a_{1}-c_{2}$, which we denote by $\delta$. Evidence for this is provided by the following, which combines Theorem 3.6 and Lemma 3.7 from [18].

Theorem 2.1. Let $\left(n, r, c_{2}\right)$ be a parameter set satisfying conditions (F1) and (F2). If $\delta=0$ then (F3) is satisfied, if $\delta= \pm 2$ then (F3) is satisfied if and only if $n$ is square. For fixed $r$ and $\delta$, there are only finitely many parameter sets satisfying (F1), (F2) and (F3), unless $\delta=-2,0$ or 2 . 


\section{$\S 3 . \quad$ Examples}

In this section we provide a number of examples of antipodal distance-regular covers of complete graphs.

Let $H$ be the Hoffman-Singleton graph. This a 7-regular graph on 50 vertices with girth five and diameter two, and this implies each path of length three in $H$ is contained in a unique 5-cycle. Let $u$ be a fixed vertex in $H$ and let $v_{1}, \ldots, v_{7}$ be the seven neighbours of $u$. Let $Y$ be the graph formed by the 42 vertices at distance two from $u$. Because $X$ has diameter two and contains no 4-cycles, each vertex in $Y$ is adjacent to exactly one of the neighbours of $u$. Therefore $Y$ is regular with valency six. If two vertices in $Y$ are adjacent to the same neighbour of $u$ then they must lie at distance at least three in $Y$, or we would have a triangle or a 4 -cycle in $X$.

Let $F_{i}$ denote the set of six vertices in $Y$ adjacent to $v_{i}$. If $x$ in $H$ is adjacent to $v_{i}$ and $j \neq i$ then $\left(x, v_{i}, u, v_{j}\right)$ is a path of length three; the fifth vertex $y$ in the unique 5 -cycle that contains it must lie in $F_{j}$. This shows that a vertex in $F_{i}$ has exactly one neighbour in $F_{j}$. Accordingly $Y$ is a covering graph of a 6-regular graph on seven vertices, necessarily $K_{7}$, with the sets $F_{i}$ as fibres. With more effort, it can be shown that this is an antipodal distance-regular cover. Conversely, any antipodal distance-regular 6-fold cover of $K_{7}$ is the set of vertices at distance from a fixed vertex in a copy of the Hoffman-Singleton graph.

A Moore graph is a graph with diameter $d$ and girth $2 d+1$. From (F1) we know that if an $\left(n, r, c_{2}\right)$-cover exists then $r-1 \leq n-2$. If equality holds, $c_{2}=1$ and so, using (2.1), we also have $a_{1}=0$. Gardiner [12] noted that an antipodal distance-regular $(n-1)$-fold cover of $K_{n}$ is equivalent to the existence of a Moore graph with diameter two and valency $n$. (So we would very much like to have a $(57,56,1)$-cover!)

Next we consider regular two-graphs. These have a fairly extensive literature. A good access point is provided by Seidel's selected works [25], which contains a number of papers on them, including two surveys.

A regular two-graph is essentially just an antipodal distance-regular cover of $K_{n}$ with index two. To introduce them, we first consider arbitrary 2-fold covers of $K_{n}$. Let $f$ be an arc-function of index two on $K_{n}$. Then, for any arc $(i, j)$ we have $f(i, j)=f(j, i)$ and so $f$ is determined by specifying the edges of $K_{n}$ on which it takes the value -1 . This set of edges is just a graph, which we will denote by $G(f)$. As noted in the introduction, we may always assume that an arc-function is equal to the identity on some spanning forest. In $K_{n}$ choose $T$ to be a copy of $K_{1, n-1}$ and only use arc-functions that are equal to the identity on $T$; this means that $G(f)$ will have an isolated vertex. More thought reveals 
that the subgraph obtained from $G(f)$ by deleting this isolated vertex is isomorphic to the neighbourhood of a vertex in $K_{n}^{f}$.

Thus covers of $K_{n}$ with index two can be specified by giving the neighbourhood of some vertex in the cover. It is not hard to see that a 2-fold cover of $K_{n}$ is distance-regular if each vertex neighbourhood is a regular graph and, if distance-regular, it is antipodal. Although any graph on $n-1$ vertices can occur as the neighbourhood of a vertex in 2 -fold cover of $K_{n}$, not all regular graphs can occur as vertex neighbourhoods in distance-regular 2 -fold covers.

Lemma 3.1. A graph $X$ can be the neighbourhood of a vertex in a distance regular double cover of a complete graph if and only it is empty, or is strongly regular with $k=2 c_{2}$.

If we take $X$ to be complete on $r$ vertices, the corresponding 2 -fold cover is two disjoint copies of $K_{n}$. This is not distance-regular, because it is not connected. If $X$ is empty then the cover is the graph obtained by deleting a perfect matching from a complete bipartite graph $K_{n, n}$. We call this a trivial distance regular cover of $K_{n}$.

The cycle on five vertices has $k=2$ and $c_{2}=1$; the corresponding double cover is the icosahedron. If $X$ is the line graph of $K_{3,3}$ then $k=4$ and $c_{2}=2$, this provides a non-trivial double cover of $K_{10}$. It can be shown that if a non-trivial distance-regular double cover of $K_{n}$ exists then $n$ must be even.

If $X$ is strongly regular and $k=2 c_{2}$ then the same relation holds for the complement $\bar{X}$ of $X$. This gives a second distance-regular double cover of $K_{n}$. The sum of the values of $\delta$ for these two covers is zero, and if the first has non-trivial eigenvalues $\theta$ and $\tau$, the second has $-\theta$ and $-\tau$. We refer to these two graphs as a complementary pair. There does not appear to be anything analogous to this when $r>2$.

A set $\mathcal{L}$ of $m$ lines in $\mathbf{R}^{n}$ is equiangular if the angle between any two distinct lines is the same. Lemmens and Seidel [20] (or [25]) show that, if the cosine of this angle is $\alpha$ and $n \alpha^{2}<1$, then

$$
m \leq \frac{n\left(1-\alpha^{2}\right)}{1-n \alpha^{2}}
$$

Equality holds if and only if there is a distance-regular 2-fold cover of $K_{n}$.

If $u$ is a vertex in the graph $X$, let $X(u)$ denote the neighbourhood of $u$ in $X$. Let $X$ be an $\left(n, r, c_{2}\right)$ cover with $\delta=0$. Then any two vertices in different fibres have the same number of common neighbours, $a_{1}=c_{2}$. Define an incidence structure $\mathcal{D}$ with the vertices of $X$ as its points and 
the sets $X(u)$, for $u$ in $V(X)$, as its blocks. Then $\mathcal{D}$ is a square divisible design and the map $\sigma$ such that $u^{\sigma}=X(u)$ is a polarity. The classes are the fibres of $X$, so two points in the same class do not lie in any block. Two vertices in different classes lie in exactly $a_{1}$ blocks.

If $X$ is a $\left(n, n-2, c_{2}\right)$-cover then (F1) implies that $c_{2}=1$ and then (2.1) yields that $a_{1}=1$. Hence $\delta=0$, and we can construct a divisible design $\mathcal{D}$. Extend $\mathcal{D}$ to a new incidence structure $\mathcal{D}^{\prime}$, as follows. Adjoin $n+1$ points $p_{1}, \ldots, p_{n}$ together with a point $\infty$. Let $F_{1}, \ldots, F_{n}$ be the fibres of $X$. Define $\ell_{\infty}=\left\{p_{1}, \ldots, p_{n}\right\}$ to be one new block, along with the blocks

$$
\ell_{i}=\infty \cup p_{i} \cup F_{i}, \quad i=1, \ldots, n .
$$

Then $\mathcal{D}^{\prime}$ has $n^{2}-n+1$ points, $n^{2}-n+1$ lines and every pair of distinct points lies on exactly one line, and we have constructed a projective plane of order $n-1$. The polarity $\sigma$ extends to a polarity of $\mathcal{D}^{\prime}$ if we define $\sigma(\infty)=\ell_{\infty}$ and $\sigma\left(\ell_{i}\right)=p_{i}$. Thus our plane has a polarity with $n$ collinear absolute points; this implies that $n-1$ is even. Conversely, every projective plane of even order $n$ that has a polarity with exactly $n$ absolute points, all collinear, determines an $(n, n-2,1)$-cover. This construction is ascribed by Biggs [1] to Bondy, who remembered none of it when I spoke to him about it. (Which is not to say that I doubt the attribution!)

The line graph of the Petersen graph is a $(5,3,1)$-cover; if we carry out the construction just described we get the projective plane of order four.

Covers with $\delta=-2$ also lead to divisible designs. We still take the vertices of $X$ to be the points of the design, this time the blocks are the sets $u \cup X(u)$. If $u^{\sigma}:=u \cup X(u)$ then $\sigma$ is, once more, a polarity. The divisible designs here are transversal designs, each block contains exactly one point from each class.

If $A$ is the adjacency matrix of an $r$-fold cover of $K_{n}$ and $\delta=-2$ then

$$
\left(\begin{array}{cc}
0 & A+I \\
A+I & 0
\end{array}\right)
$$

can be shown to be the adjacency matrix of an antipodal distance-regular $r$-cover of $K_{n, n}$. Distance-regular covers of complete bipartite graphs are another interesting class of objects; for more information about them, see [13].

If $X$ is an $\left(n, r, c_{2}\right)$-cover with $\delta=2$ we can, once again, construct a divisible design from it. Let $X_{i}$ denote the graph with the same vertex set as $X$, where two vertices are adjacent if and only if they are at distance $i$ in $X$. Since $X$ is antipodal, $X_{3}$ consists of $n$ vertex disjoint 
copies of $K_{r}$. Let $A_{i}$ be the adjacency matrix of $X_{i}$. Then $A_{1}=A$ (in our usual notation) and, as

$$
A_{1}^{2}=(n-1) I+a_{1} A_{1}+c_{2} A_{2} .
$$

If we substitute $J-I-A_{1}-A_{3}$ for $A_{2}$ and observe that $A_{2}=A_{1} A_{3}$, this implies that

$$
\left(A_{1}+A_{3}\right)^{2}=\left(n-c_{2}+r-4\right) I+\left(c_{2}+2\right)\left(J-A_{3}\right)+(r-2) A_{3} .
$$

Therefore $A_{1}+A_{3}$ is the incidence matrix of a square divisible design (with a polarity). When $r=c_{2}+4$ we actually get a symmetric design, but in this case we have stumbled onto a known construction of symmetric designs from a generalised quadrangle with parameters $(q+1, q-1)$. (We will see in the next section that covers such that $\delta=2$ and $r=c_{2}+4$ can be built from a $G Q(q+1, q-1)$ with a spread.) For more information on these designs, see [23, Ch. 3.6].

The possible parameter sets of a square group divisible design are constrained by the Bose-Connor conditions [2]. These imply that covers with parameter sets $(11,3,3)$ and $(7,5,1)$ do not exist. The latter would, of course, give rise to a projective plane of order six. The Bose-Connor conditions only provide extra information when $\delta=0$; if $\delta= \pm 2$ then we already have that $n$ must be a perfect square.

\section{$\S 4 . \quad$ Constructions}

We will describe some of the main techniques for constructing families of distance-regular covers of $K_{n}$. The relations between the various known classes of covers are discussed in [5], which might even appear one day.

Standard Covers: We call a cover standard if $\delta=0$. We present a construction of a class of standard covers due to Neumaier, as described in $[4,12.5 .3]$. The graphs produced were first found by Mathon, using a different technique. Let $q$ be a prime power, let $K$ be the subgroup of $r$-th powers in the multiplicative group of $F$ and suppose that $-1 \in K$. Let $V$ be a vector space of dimension two over $F$, provided with a nondegenerate symplectic form $B$. Let $X$ be the graph with vertices the sets $K v$, where $v$ ranges over the non-zero vectors in $V$, and where $K u$ and $K v$ are adjacent if and only if $B(u, v) \in K$. Then $X$ is a cover with $\delta=0$ and parameters $(q+1, r,(q-1) / r)$; the largest possible value of $r$ is $q-1$ when $q$ is even and $(q-1) / 2$ if $q$ is odd.

It is shown in [18] that an antipodal cover of $K_{n}$ with diameter three is distance-regular if and only if there is a constant, $\mu$ say, such 
that any two non-adjacent vertices in different fibres have exactly $\mu$ common neighbours. (If $\mu$ is well-defined then it is equal to $c_{2}$.) This is a trick that makes it easier to verify that the graphs we have just defined are distance-regular, and it can be also applied to the constructions that follow.

In [9], Peter Cameron provides a number of constructions of covers with $\delta=0$. It appears that most of these are isomorphic to quotients of the covers just described. (We will discuss the operation of taking quotients of covers towards the end of this section.) However the two classes of graphs he constructs from unitary forms cannot be obtained in this way, although in one case $\delta=0$.

Symplectic Covers: Next, let $p$ be a prime, and let $V$ and $U$ be vector spaces over $G F(p)$, with dimensions $m$ and $s$ respectively. Let $B$ be a $G F(p)$-linear alternating form from $V \times V$ to $U$. Thus, if $v$ and $w$ belong to $V$ then $B(v, w) \in U$ and

$$
B(v, v)=0, \quad B(v, w)=-B(v, w) .
$$

Assume further that, for each element $a$ in $V$, the linear mapping $B_{a}$ for $V$ to $U$ given by

$$
B_{a}(v)=B(a, v)
$$

is onto. Clearly this implies that $\operatorname{dim} U \leq \operatorname{dim} V$ and, since $B(a, a)=0$, it follows that $\operatorname{dim} U<\operatorname{dim} V$. Let $X(B)$ be the graph with vertex set $V \times U$, where $(v, a)$ and $(w, b)$ are adjacent in $X$ if and only if they are distinct and

$$
B(v, w)=a-b .
$$

A straightforward computation shows that this yields a $\left(p^{m}, p^{s}, p^{m-s}\right)$ cover. As $\delta=-2$ for this cover, $m$ must be even. If $U$ is 1-dimensional, this construction reduces to one due to Somma, that in turn generalises a construction of Thas. For more on these, see Section 12.5 of [4].

We describe a class of examples. Let $\Sigma$ be a set of invertible $m \times m$ skew-symmetric matrices over $G F(p)$ that forms a subspace over $G F(p)$. Let $A_{1}, \ldots, A_{s}$ be a basis for this subspace and define $B(v, w)$ to be the vector with entries $v^{T} A_{1} w, \ldots, v^{T} A_{s} w$. We must verify that $B_{a}$ is onto. Let $M(a)$ be the matrix with $a^{T} A_{i}(i=1, \ldots, s)$ as its rows. Then $M(a) w=B(a, w)$ and, if $x=\left(x_{1}, \ldots, x_{s}\right)$, then

$$
x^{T} M(a)=a^{T} \sum_{i=1}^{s} x_{i} A_{i} .
$$

If $x \neq 0$ then $x_{i} A_{i}$ is invertible; this implies that the rows of $M(a)$ are linearly independent. Consequently $M(a)$ has rank $s$ and $B_{a}$ is onto. 
It is not to difficult to see that changing the basis of $\Sigma$ is equivalent to replacing $B$ by a form $B^{\prime}$, where $B^{\prime}=g B$ for some invertible $s \times s$ matrix $g$ over $G F(p)$. Further $X(B)$ and $X\left(B^{\prime}\right)$ are isomorphic.

A spread set $\Sigma$ is a set of $q^{n}$ matrices of order $n \times n$ over $G F(q)$, such that the difference of any two matrices in it is invertible. Subtracting a fixed matrix from each element gives rise to a spread set that contains the zero matrix, in this case all non-zero matrices in the set are invertible. Spread sets are equivalent to translation planes; if $\Sigma$ is closed under addition then the translation plane is a semifield plane. (If $q$ is a power of the prime $p$ then $\Sigma$ is closed under addition if and only if it is a subspace over $G F(p)$.) For some more information on this subject, see [21]. Suppose then that $\Sigma$ is spread set such that is a vector space over $G F(p)$. If $S_{i} \in \Sigma$ let $\hat{S}_{i}$ be the skew symmetric matrix

$$
\left(\begin{array}{cc}
0 & S_{i} \\
S_{i}^{T} & 0
\end{array}\right)
$$

Then the matrices $\hat{S}_{i}$, form a subspace of invertible skew symmetric matrices.

De Caen (private communication) and Cameron [19, Thm. 3.8] have observed that a subspace of invertible $2 m \times 2 m$ matrices over a finite field has dimension at most $m$. This bound is realised by the semifield examples we have just described.

The graph $X(B)$ is the Cayley graph for a $p$-group. Let $P$ be the group with vertex set $V \times U$ and multiplication given by

$$
(v, a) \oplus(w, b)=(v+w, a+b+B(v, w)) .
$$

Then $P$ is elementary abelian if $p=2$, otherwise it is special and has exponent $p$. In all cases it acts as a regular group of automorphisms of $X$ and, if $p$ is odd, the fibres of $X(B)$ are the cosets of the centre of $P$.

Spreads in Generalised Quadrangles: Our third construction uses generalised quadrangles and is due to Brouwer [4, Prop. 12.5.2]. A spread in a generalised quadrangle is a set of lines that partitions its points. Let $\mathcal{Q}$ be a $G Q(s, t)$ with a spread $\Sigma$. Let $X$ be the graph with the points of $\mathcal{Q}$ as its vertices, with two points adjacent in $X$ if and only if there are collinear in $\mathcal{Q}$, but the line joining them is not in $\Sigma$. This yields an $(s t+1, s+1, t-1)$-cover.

More generally, it is possible to construct covers from strongly regular graphs that have the same parameters as the point graph of a generalised quadrangle, and have a spread. For details, see [4, Prop. 12.5.2] or [18]. Note also that a generalised quadrangle may have more than one spread, and hence may give rise to more than one cover. 
For information about generalised quadrangles and their spreads, see [23]. The parameter sets of the generalised quadrangles that are known to have spreads, and the parameters of the related covers, are listed in the following table.

\begin{tabular}{|c|c|c|}
\hline$(s, t)$ & $\left(n, r, c_{2}\right)$ & $\delta$ \\
\hline$(q, q)$ & $\left(q^{2}+1, q+1, q-1\right)$ & 0 \\
$\left(q, q^{2}\right)$ & $\left(q^{3}+1, q+1, q^{2}-1\right)$ & $q-q^{2}$ \\
$(q-1, q+1)$ & $\left(q^{2}, q, q\right)$ & -2 \\
$(q+1, q-1)$ & $\left(q^{2}, q+2, q-2\right)$ & 2 \\
\hline
\end{tabular}

Here $q$ is a prime power in the first three cases, and a power of two in the fourth.

Preparata Covers: Recently de Caen, Mathon and Moorhouse [7] found a new infinite family of covers which are related to the Preparata codes and have parameter sets $\left(2^{2 t}, 2^{2 t-1}, 2\right)$. These can be described quite succinctly. Assume $q=2^{2 t-1}$ and $s=2^{e}$, where $e$ and $2 t-1$ are coprime. Let $X(q, s)$ be the graph with vertex set

$$
G F(q) \times G F(2) \times G F(q),
$$

where $(a, i, \alpha)$ and $(b, j, \beta)$ are adjacent if and only if

$$
\alpha+\beta=a^{s} b+a b^{s}+(i+j)\left(a^{s+1}+b^{s+1}\right) .
$$

Then $X(q, s)$ is a $\left(2^{2 t}, 2^{2 t-1}, 2\right)$-cover. The fibres are the sets of the form

$$
\{(a, i, u): u \in G F(q)\} \text {. }
$$

The maps

$$
(a, i, \alpha) \mapsto(a, i, \alpha+u), \quad u \in G F(q)
$$

generate the covering group of $X(q, s)$, which is therefore regular and isomorphic to the additive group of $G F(q)$. The graphs $X\left(q, 2^{e}\right)$ and $X\left(q, 2^{f}\right)$ are isomorphic if and only if $e+f$ is zero, modulo $2 t+1$. These covers are all triangle-free; prior to their construction the only examples of triangle-free cover known were the $(7,6,1)$-cover coming from the Hoffman-Singleton graph and the cycle on six vertices.

Another feature of these covers is that corresponding association schemes are formally dual to the schemes formed by linked systems of symmetric designs. For information about the connection, see [7]; for information about these systems see, e.g., [10, p. 148].

Schur Groups: Let $f$ be an arc function of index $r$ on $K_{n}$, normalised with respect to some spanning tree and suppose that the covering group 
of $X^{f}$ is abelian. Then the connection group $C$ is abelian and has $r$ distinct irreducible characters $\chi_{1}, \ldots, \chi_{r}$, where $\chi_{1}$ is the trivial character. Let $H_{s}$ be the $n \times n$ matrix with diagonal entries equal to 0 and

$$
\left(H_{s}\right)_{i, j}=\chi_{s}(f(i, j)) .
$$

The set of matrices $\mathcal{H}=\left\{H_{1}, \ldots, H_{r}\right\}$ has the following properties:

(a) $H_{1} \circ H_{i}=H_{i}$ for all $i$.

(b) $H_{i}^{*}=H_{i}$.

(c) $H_{i} \circ H_{j} \in \mathcal{H}$, for all $i$ and $j$.

(d) The adjacency matrix $A$ of $X^{f}$ is similar to a block-diagonal matrix, with $H_{1}, \ldots, H_{r}$ as its diagonal blocks.

Here $A \circ B$ denotes the $S c h u r$ or pointwise product of matrices $A$ and $B$. A set of $r$ matrices satisfying (a) and (c) is called a Schur group, because it is closed under Schur multiplication. Note the entries of the matrices in a Schur group of order $r$ must be $r$-th roots of unity. As the irreducible characters of an abelian group $C$ form a multiplicative group isomorphic to $C$, the Schur group of a cover with regular abelian covering group is isomorphic to the covering group.

Now suppose that $X$ is an $\left(n, r, c_{2}\right)$-cover with abelian connection group, and Schur group $\mathcal{H}=\left\{H_{1}, \ldots, H_{r}\right\}$. Then $H_{1}=J$ and, when $2 \leq i \leq r$,

$$
H_{i}^{2}-\delta H_{i}-(n-1) I=0 .
$$

Assume further that the covering group of $X$ has exponent two and $i \geq 2$. If $\delta=0$, then (4.1) implies that $H_{i}$ is a conference matrix for $i=2, \ldots, r$. If $\delta=-2$ then $H_{i}+I$ is a Hadamard matrix, and the matrices $H_{i}+I$ form a Schur group with identity element $J$. Finally, if $\delta=2$ then $H_{i}-I$ is a Hadamard matrix. (If we drop the assumption that the covering group has exponent two, "generalised" Hadamard matrices result.)

The converse is also true, and is proved in Section 9 of [18].

Theorem 4.1. Let $\mathcal{H}$ be a Schur group of $n \times n$ Hermitian matrices with order $r$, such that

$$
H_{i}^{2}-\delta H_{i}-(n-1) I=0
$$

when $2 \leq i \leq r$. Then $\mathcal{H}$ determines an $\left(n, r, c_{2}\right)$-cover of $K_{n}$ with cover group isomorphic to $\mathcal{H}$.

By way of example, let $q$ be a power of the prime $p$, let $V$ be a vector space of dimension $2 m$ over $G F(q)$, let $A$ be a non-degenerate 
alternating bilinear form on $V$ and let $\xi$ be a primitive $q$-th root of unity. Let $H(A)$ be the matrix with rows and columns indexed by the entries of $V$, such that

$$
H(A)_{u, v}:=\xi^{u^{T} A v} .
$$

Then $H_{A}^{2}=q^{2 m} I$ and, if $B$ is a second alternating form, then

$$
H(A) \circ H(B)=H(A+B) .
$$

It follows that, if $S$ is subspace of invertible skew-symmetric matrices over $G F(p)$, then the matrices $H(S)$ form a Schur group.

The Preparata covers have a regular covering group that is an elementary abelian 2 -group. Hence they give rise to a Schur group of $2^{2 n-1}$ symmetric $2^{2 n} \times 2^{2 n}$ Hadamard matrices with constant diagonal.

If $\mathcal{H}=\left\{H_{1}, \ldots, H_{r}\right\}$ is the Schur group of an $\left(n, r, c_{2}\right)$-cover $X$ and $i \neq 1$ then $H_{i}$ determines a distance-regular cover, $X_{i}$ say, of $K_{n}$, with index equal to the order of $H_{i}$. It follows that $X$ can be expressed as the product of distance-regular covers of $K_{n}$, each with regular cyclic covering group. The condition that the matrices $H_{i}$ form a Schur group is equivalent to the condition that the product of the covers $X_{i}$ be distanceregular. Note that any cover with regular abelian covering group can always be expressed as a product of covers with cyclic covering groups.

Quotients: There is a useful technique which often enables us to construct covers of lower index from a given cover of index $r$. We describe a special case. Let $X$ be an $r$-fold cover of $K_{n}$ and let $H$ be a group of automorphisms of $X$ that fixes each fibre as a set. As noted before, $H$ must act semi-regularly and so $|H|$ must divide $r$. Construct a graph with the orbits of $H$ as its vertices, with two orbits adjacent if and only if some vertex in one is adjacent to a vertex in the second. It is easy to show that this new graph is a cover of $K_{n}$ with index $r /|H|$; it can also be shown that is antipodal and distance regular. For details, see Section 6 of [18]. The quotienting procedure just described can be generalised. The only role $H$ plays in the construction is to provide a nice partition of the vertex set of $X$. Any equitable partition of $X$ with each cell contained in a fibre of $X$ is "nice" enough. For details, see Section 6 of [18]. In all our applications, we will use the group version.

If $X$ is an $\left(n, r, c_{2}\right)$-cover and $h=|H|$ then the quotient of $X$ over the orbits of $H$ is a $\left(n, r / h, h c_{2}\right)$-cover. This means that the value of $\delta$ is not changed when we form quotients.

But which of our examples admit quotients? Most! The covering groups of the standard covers obtained from Neumaier's construction are regular and cyclic, being quotient groups of the multiplicative group of 
the underlying field. This set of covers is closed under taking quotients. The covering groups of the symplectic covers are regular and elementary abelian and, once again, this class is closed under taking quotients. The covering groups of the Preparata covers of are regular elementary abelian 2-groups. In this we obtain new covers by taking quotients. (To be more precise, any quotient with $r^{2}>n$ has not been constructed in any other way.) The unitary generalised quadrangles, with parameters $\left(q, q^{2}\right)$, give rise to covers with regular cyclic covering group. The corresponding quotients were first found by Cameron [9]. (For more on these, see also [16].) We cannot realise any new parameter sets by taking quotients of the covers coming from generalised quadrangles with parameters $(q, q)$ and $(q-1, q+1)$. I do not if the covers coming from generalised quadrangles with parameters $(q+1, q-1)$ have any quotients; it will follow from our next theorem that these covers cannot be abelian.

Suppose $S$ is the Schur group determined by an $\left(n, r, c_{2}\right)$-cover with abelian regular covering group $C$. Then any non-identity subgroup of $S$ determines a cover with index equal to its order. The Schur group is isomorphic to the dual group of $C$, hence its subgroups correspond to homomorphic images of $C$. This implies that taking the cover formed from a Schur subgroup is equivalent to forming a quotient of the cover.

In combination with taking quotients, the following result is often useful.

Theorem 4.2 ([18, Thm. 9.2]). Let $X$ be an antipodal distanceregular cover of $K_{n}$ with index $r$, and suppose that the covering group of $X$ is regular and cyclic. If $X$ is not a trivial 2 -fold cover of $K_{n}$, then $r$ divides $n$.

For example, this can be used to show that the covering group of a 6 -fold cover of $K_{7}$ is trivial. Suppose that $C$ is the covering group of such a cover $X$. Then $X$ has parameters $(7,6,1)$; hence $\delta=-1$. If $C$ has a subgroup of order two then, quotienting over this yields a $(7,3,2)$ cover while, if $C$ has a subgroup of order three, quotienting yields a $(7,2,3)$-cover. In both cases we find that the eigenvalue multiplicities are not integers, hence these quotients do not exist. Because $C$ has to act semi-regularly on a fibre, $|C|$ must divide six. Hence we have shown that $C$ must be trivial. (Or, to put it another way, the stabiliser of a vertex in the Hoffman-Singleton graph acts faithfully on the neighbours of that vertex.)

There is a 4-fold cover of $K_{10}$ with regular abelian covering group; this shows that Theorem 4.2 does not hold if we replace "cyclic" by "abelian". 


\section{$\S 5$. Linear association schemes}

A graph $X$ is called a translation graph if there is an abelian group of automorphisms acting regularly on its vertices. For our purposes the most useful abelian groups are the additive groups of a vector space $V=V(n, q)$ over a field $F$ of order $q$. We can construct graphs relative to $V$ as follows. Let $\mathcal{C}$ be a subset of non-zero vectors in $V$ that is closed under multiplication by -1 . Then $X(\mathcal{C})$ is the graph with vertex set $V$, and vectors $u$ and $v$ are adjacent in $x$ if and only if $u-v \in \mathcal{C}$. Thus $X$ is a Cayley graph for the additive group of $V$. We say that $X(\mathcal{C})$ is linear over $F$ is $\mathcal{C}$ is closed under multiplication by the non-zero elements of $F$.

There is an attractive geometric construction for linear graphs. Let $\mathcal{P}$ be a hyperplane in $n$-dimensional projective space $P G(n, q)$, and view $\mathcal{P}$ as the hyperplane at infinity for the affine space $A G(n, q)$ formed by the points not on $\mathcal{P}$. Choose a subset $\Omega$ in $\mathcal{P}$ and define $X(\Omega)$ to be the graph with $A G(n, q)$ as its vertex set, where two points $u$ and $v$ are adjacent if the line $u \vee v$ meets $\mathcal{P}$ at a point in $\Omega$. The correspondence with the previous description is trivial: the subset $\Omega$ determines a set of lines through the origin in $A G(n, q)$, and the non-zero points in these lines form the set $\mathcal{C}$. The valency of $X(\Omega)$ is $(q-1)|\Omega|$, and this one of its eigenvalues. The remaining eigenvalues of $X(\Omega)$ are also determined geometrically by the following result from [15, Lemma 12.9.3].

Lemma 5.1. Let $X(\Omega)$ be a linear graph, where $\Omega$ is a subset of the projective space $\mathcal{P}$ over $G F(q)$, and let $n_{x}$ denote the number of hyperplanes of $\mathcal{P}$ that meet $\Omega$ in exactly $x$ points. Then $q x-|\Omega|$ is an eigenvalue of $X$ with multiplicity $(q-1) n_{x}$.

We need yet a third version of our construction. Let $C$ be a linear code in $V(n, q)$. The coset graph of $C$ has the cosets of $C$ as its vertices, where two cosets are adjacent if there is a vector in one at Hamming distance one from a vector in the second. We may prevent multiple edges occurring by assuming that the minimum distance of $C$ is at least three. It is an easy exercise to show that the coset graph of a linear code with minimum distance three is linear. The following result is essentially Theorem 11.1.10 from Brouwer, Cohen and Neumaier [4].

Theorem 5.2. Let $X$ be a distance-regular graph that is not a self-complementary conference graph. If $X$ is a translation graph with respect to an elementary abelian p-group then it is the coset graph of a completely regular linear code with minimum distance at least three. 
Because we will only apply this result to graphs with diameter three, all we need to say about conference graphs is that they have diameter two. And it will suffice to define a linear code to be completely regular if its coset graph is distance-regular. (For more information, see Chapter 11 in either [4] or [15].) Our application of Theorem 5.2 is as follows. Suppose $X$ is an antipodal distance-regular graph that is linear over $G F\left(p^{s}\right)$, and let $G$ be a proper, non-identity, subgroup of the covering group of $X$. As $X$ is linear, there is an elementary abelian $p$-group, $T$ say, acting regularly on $X$. The fibres of $X$ are a system of imprimitivity for $T$ and, as $T$ is abelian, the subgroup of $T$ fixing each fibre is non-trivial and acts regularly on the vertices in a fibre. Thus this subgroup is the covering group of $X$. Accordingly, if $G$ is a subgroup of the covering group of $X$ then the quotient of $X$ over the partition formed by the orbits of $G$ is antipodal and distance-regular. It is also clearly a translation graph for the elementary abelian quotient group $T / G$, whence we deduce, using Theorem 5.2 , that it is linear over some finite field of characteristic $p$. (Although possibly not over the field we started with.)

We turn to linear association schemes. We may view an association scheme with $d$ classes on the vertex set $V$ as a set of graphs $X_{1}, \ldots, X_{d}$ on $V$ whose edges partition the edge set of the complete graph on $V$. We call an association scheme linear if each graph in it is linear, relative to the same vector space of course, Therefore a linear association scheme is determined by giving a partition of the points of a projective space. But then we are faced with the question: which partitions give rise to association schemes? There is a nice answer to this.

Now suppose that $\omega=\left(\Omega_{1}, \ldots, \Omega_{d}\right)$ is a partition of the points of $\mathcal{P}$ with $d$ cells. Define two hyperplanes $H$ and $K$ of $\mathcal{P}$ to be $\omega$-equivalent if

$$
\left|H \cap \Omega_{i}\right|=\left|K \cap \Omega_{i}\right|, \quad i=1, \ldots, d .
$$

This gives rise to a partition of the hyperplanes of $\mathcal{P}$ into $\omega$-equivalence classes, that we will call the partition induced by $\omega$.

For a proof of the next result, due to Bridges and Mena, see [3] or [15, Thm. 12.10.1].

Theorem 5.3. Let $\mathcal{P}$ be the projective space formed by the points at infinity of an affine space of dimension $n$ over $G F(q)$. Let $\omega=$ $\left(\Omega_{1}, \ldots, \Omega_{d}\right)$ be a partition of the points of $\mathcal{P}$ and let $\omega^{*}$ be the induced partition on the hyperplanes of $\mathcal{P}$. Then $\omega^{*}$ has at least as many cells as $\omega$ and, equality holds if and only if the graphs $X\left(\Omega_{1}\right), \ldots, X\left(\Omega_{d}\right)$ form an association scheme with $d$ classes. 
Call a partition $\omega$ of the points of $\mathcal{P}$ equitable if the dual partition $\omega^{*}$ has the same number of cells as $\omega$. If $\omega$ is equitable then it follows (see, e.g., [15, p. 250]) that $\omega$ is the partition induced by $\omega^{*}$ on the hyperplanes of the space dual to $\mathcal{P}$. Hence $\omega^{*}$ determines a second association scheme that is dual, in the sense of Delsarte $[11, \S 2.6]$, to the scheme determined by $\omega$. (We discuss duality in a little more detail in Section 7.) The hyperplanes in a given cell $\Omega_{i}^{*}$ of $\omega^{*}$ determine an eigenspace with dimension $(q-1)\left|\Omega_{i}^{*}\right|$ for the association scheme given by $\omega$.

As one example, we may take $\omega$ to be the orbits of a group $\Gamma$ of collineations of $\mathcal{P}$; this is always equitable. The cells of $\omega^{*}$ are the orbits of $\Gamma$ on hyperplanes.

Let $\Omega_{1}, \ldots, \Omega_{d}$ be a partition of the points of $\mathcal{P}$ and let $X_{i}$ denote $X\left(\Omega_{i}\right)$. If $a$ and $b$ are affine points, let $p_{i, j}(a, b)$ denote the number of affine points $x$ such that $a$ is adjacent to $x$ in $X_{i}$ and $b$ is adjacent $x$ in $X_{j}$. Then the line $a \vee x$ must meet $\mathcal{P}$ in a point $p$ of $\Omega_{i}$, while $b \vee x$ meets it in a point $q$ of $\Omega_{j}$. As $a, b$ and $x$ are coplanar, $p, q$ and the point $z=(a \vee b) \cap \mathcal{P}$ must be collinear. It follows that, if $i \neq j$ and $z$ does not lie in $\Omega_{i}$ or $\Omega_{j}$, then

$$
p_{i, j}(a, b)=\sum_{\ell}\left|\ell \cap \Omega_{i}\right|\left|\ell \cap \Omega_{j}\right|
$$

where the sum is over all lines in $\mathcal{P}$ on $z$. If $\omega$ is equitable then $X_{1}, \ldots, X_{d}$ form an association scheme, and the value $p_{i, j}(a, b)$ is determined by the index $k$ such that $\{a, b\}$ is an edge of $X_{k}$. In this case the sum in (5.1) is independent of the choice of $z$ in $\Omega_{k}$, and therefore it is equal to

$$
\left|\Omega_{k}\right|^{-1} \sum_{\ell \in \mathcal{P}}\left|\ell \cap \Omega_{i}\right|\left|\ell \cap \Omega_{j}\right|\left|\ell \cap \Omega_{k}\right| .
$$

If $\omega$ is equitable and $\{a, b\}$ is an edge of $X_{k}$ then we write $p_{i, j}(k)$ in place of $p_{i, j}(a, b)$. These are the intersection numbers of the association scheme.

Theorem 5.4. Let $\mathcal{P}$ be a hyperplane in a projective space over $G F(q)$, let $\omega$ be an equitable partition of $\mathcal{P}$ with cells $\Omega_{1}, \ldots, \Omega_{d}$. Assume $i, j$ and $k$ are distinct and non-zero. Then the intersection numbers $p_{i, j}(k)$ are given as follows.
(a) $p_{i, j}(0)=\delta_{i j}(q-1)\left|\Omega_{i}\right|$.
(b) $p_{i, j}(k)=\left|\Omega_{k}\right|^{-1} \sum_{\ell \in \mathcal{P}}\left|\ell \cap \Omega_{i}\right|\left|\ell \cap \Omega_{j}\right|\left|\ell \cap \Omega_{k}\right|$.
(c) $p_{i, i}(k)=\left|\Omega_{k}\right|^{-1} \sum_{\ell \in \mathcal{P}}\left(\left|\ell \cap \Omega_{i}\right|-1\right)\left|\ell \cap \Omega_{i}\right|\left|\ell \cap \Omega_{k}\right|$.
(d) $p_{k, k}(k)=q-2+\left|\Omega_{k}\right|^{-1} \sum_{\ell \in \mathcal{P}}\left|\ell \cap \Omega_{k}\right|\left(\left|\ell \cap \Omega_{k}\right|-1\right)\left(\left|\ell \cap \Omega_{k}\right|-2\right)$. 
An association scheme $X_{1}, \ldots, X_{d}$ is primitive if each graph $X_{i}$ is connected. If this scheme is given by an equitable partition $\omega$ of $\mathcal{P}$, it is easy to show that it is primitive if and only if each cell of $\omega$ spans $\mathcal{P}$. In particular, $X(\Omega)$ is a disjoint union of cliques if and only if $\Omega$ is a subspace of $\mathcal{P}$.

Linear association schemes are discussed at some length in Chapter 12 of [15].

\section{§6. Linear covers}

We are going to study linear antipodal distance-regular covers of $K_{n}$. To begin, we present a class of examples due to Thas. (For further information, see $[4, \S 12.5]$.) Let $\mathcal{P}$ be a hyperplane in $P G(3, q)$, where $q$ is even. Let $\Omega_{1}$ be an oval in $\mathcal{P}$, that is, a set of $q+1$ points with no three collinear. Let $\Omega_{3}$ be the nucleus of the oval, and let $\Omega_{2}$ be the remaining points. We claim that $X\left(\Omega_{1}\right)$ is a $\left(q^{2}, q, q\right)$-cover. This will follow readily from Theorem 5.4 if we show that our partition is equitable. But this is easy. All lines on the nucleus meet the oval in exactly one point. Any line not on the nucleus is not a tangent, hence it is either a secant, meeting the oval in exactly two points, or a passant with no point in common with the oval. Thus the induced partition on lines has exactly three classes, and therefore $\left(\Omega_{1}, \Omega_{2}, \Omega_{3}\right)$ is equitable.

The fibres of the cover we get are the sets of points on affine lines that meet the nucleus of the oval. The transvections in $P G(3, q)$ with axis $\mathcal{P}$ and $\Omega_{3}$ as centre form the covering group, which is thus regular and isomorphic to the additive group of $G F(q)$. Hence we may take quotients and obtain covers with parameters $\left(q^{2}, q / t, q t\right)$, for any divisor $t$ of $q$.

Our next task is to characterise the equitable partitions of projective space that give rise to such graphs.

Theorem 6.1. Let $\mathcal{P}$ be a hyperplane in $P G(n, q)$ and let $\omega=$ $\left(\Omega_{1}, \Omega_{2}, \Omega_{3}\right)$ be an equitable partition of $\mathcal{P}$. Then $X\left(\Omega_{1}\right)$ is an antipodal distance-regular cover of a complete graph if and only if:

(a) $\Omega_{1}$ is not a subspace,

(b) any line of $\mathcal{P}$ that meets $\Omega_{1}$ in two or more points is disjoint from $\Omega_{3}$ and

(c) $\Omega_{3}$ is a subspace.

Proof. Because $\omega$ is equitable, the graphs $X\left(\Omega_{i}\right)$ do form an association scheme $\mathcal{A}$. As $\Omega_{1}$ is not a subspace, there is line $\ell$ such that $\left|\ell \cap \Omega_{1}\right| \geq 2$ but $\ell$ is not contained in $\Omega_{1}$. From condition (b) it follows that $\ell \cap \Omega_{2} \neq \emptyset$, and now Theorem 5.4 yields that $p_{1,1}(2)>0$. Now 
choose $\ell$ to be a line meeting both $\Omega_{1}$ and $\Omega_{3}$. Then (b) implies that $\ell$ intersects $\Omega_{1}$ in exactly one point, and (c) implies that it intersects $\Omega_{3}$ in exactly one point. Therefore $\ell$ must contain at least one point from $\Omega_{2}$, whence Theorem 5.4 yields that $p_{1,2}(3)>0$. This implies that $X\left(\Omega_{1}\right)$ has diameter three, and consequently $\mathcal{A}$ is metric relative to $X\left(\Omega_{3}\right)$, that is, $X\left(\Omega_{3}\right)$ is distance regular.

Because $\Omega_{3}$ is a subspace, being "equal or at distance three" in $X\left(\Omega_{1}\right)$ is an equivalence relation on its vertices. Therefore $X$ is antipodal with diameter three, and must be an antipodal cover of a complete graph.

Q.E.D.

Suppose $\Omega_{3}$ has dimension $t$. Then condition (b) in the theorem is equivalent to the condition that each $(t+1)$-dimensional subspace of $\mathcal{P}$ on $\Omega_{3}$ must intersect $\Omega_{1}$ in exactly one point. A subspace $U$ of $\mathcal{P}$ is a complement to a subspace $V$ if it is disjoint from it and

$$
\operatorname{dim} U+\operatorname{dim} V=\operatorname{dim} \mathcal{P} .
$$

If $U$ is a complement to $V$ then the partition of $\mathcal{P}$ with cells $U, V$ and $\mathcal{P} \backslash(U \cup V)$ is equitable. Further any line that meets $U$ in at least two points is contained in $U$, and consequently is disjoint from $V$. If $V$ is a subspace of $\mathcal{P}$, define a subset $\Omega$ of $\mathcal{P}$ to be a pseudo-complement for $V$ if:

(1) The partition $(\Omega, V, \mathcal{P}-(\Omega \cup V))$ is equitable.

(2) Any line that meets $\Omega$ in at least two points is disjoint from $V$.

Then Theorem 6.1 implies that if $\Omega_{3}$ is a subspace of $\mathcal{P}$ and $\Omega_{1}$ is a pseudo-complement to it then it is a subspace of $X\left(\Omega_{1}\right)$ is an antipodal distance-regular graph with diameter three.

Suppose that $\mathcal{P}$ is a hyperplane in $P G(d+1, q),\left(\Omega_{1}, \Omega_{2}, \Omega_{3}\right)$ is an equitable partition of $\mathcal{P}$ such that $\Omega_{3}$ is a subspace of dimension $t$ and $\Omega_{1}$ is a pseudo-complement to $\Omega_{3}$ that is not a subspace. Then

$$
\begin{aligned}
& \left|\Omega_{1}\right|=\frac{q^{d-t}-1}{q-1} \\
& \left|\Omega_{2}\right|=(q-1) \frac{q^{d-t}-1}{q-1} \frac{q^{t+1}-1}{q-1} \\
& \left|\Omega_{3}\right|=\frac{q^{t+1}-1}{q-1}
\end{aligned}
$$

Further $X\left(\Omega_{1}\right)$ is a cover of a complete graph, having parameters

$$
n=q^{d-t}, \quad r=q^{t+1} .
$$


As $r \leq n-1$, we see that

$$
d \geq 2 t+2 .
$$

In the next section we will find more restrictions on the parameters of a linear cover.

\section{$\S 7 . \quad$ Parameters of linear covers}

Assume $X_{1}, \ldots, X_{d}$ form an association scheme $\mathcal{A}$ on $n$ vertices. The valency of $X_{i}$ is denoted by $v_{i}$. Set $v_{0}$ equal to 1 . The Bose-Mesner algebra of the scheme has $d+1$ distinct eigenspaces, with dimensions $m_{0}, \ldots, m_{d}$, where $m_{0}=1$. These are the multiplicities of the scheme. The Frame quotient of the scheme is defined to be

$$
n^{d-1} \prod_{i=1}^{d} \frac{v_{i}}{m_{i}} .
$$

It is always an integer. (For details, see [4, p. 46].) Our scheme has a $(d+1) \times(d+1)$ matrix of eigenvalues $P$, and a matrix of dual eigenvalues $Q$, of the same order, and $P Q=n I$. A second association scheme is formally dual to $\mathcal{A}$ if its matrix of eigenvalues is $Q$; this scheme has valencies $m_{0}, \ldots, m_{d}$ and multiplicities $v_{0}, \ldots, v_{d}$.

If $\mathcal{A}$ is a translation scheme then we may identify its vertices with the elements of an abelian group $G$. In this case there is a well-defined construction of a dual scheme, with the irreducible characters of $G$ as its vertices. A dual scheme is formally dual in the sense just defined. If $\omega$ is an equitable partition of a projective space with induced partition $\omega^{*}$ then the schemes determined by $\omega$ and $\omega^{*}$ are dual, so all linear schemes have duals. (For more background on duality, see $[4, \S 2.10(\mathrm{~B})]$, $[15$, Ch. 12] and $[11, \S 2.6]$.)

Now we are in a position to prove the main result of this section.

Theorem 7.1. Let $X$ be an $\left(n, r, c_{2}\right)$-cover with $p^{a}$ vertices, where $p$ is a prime, and suppose there is an association scheme that is formally dual to the scheme determined by $X$. Then $p=2, n$ is a perfect square and either $\delta=-2$ or $r=2$ and $\delta=2$.

Proof. Let $\theta$ and $\tau$ be the non-trivial eigenvalues of $X$. We first prove that $\theta-\tau$ divides $n$, which implies that it is power of $p$.

We compute the Frame quotient $F^{*}$ of the formally dual scheme. We have

$$
m_{\theta} m_{\tau}=-\frac{n^{2}(r-1)^{2} \theta \tau}{(\theta-\tau)^{2}}=\frac{n^{2}(r-1)^{2}(n-1)}{(\theta-\tau)^{2}} .
$$


and so

$$
\begin{aligned}
F^{*} & =(n r)^{2} \frac{(n-1) m_{\theta} m_{\tau}}{(n-1) \cdot(r-1)(n-1) \cdot(r-1)} \\
& =(n r)^{2} \frac{n^{2}(r-1)^{2}(n-1)}{(r-1)^{2}(n-1)(\theta-\tau)^{2}} \\
& =\frac{n^{4} r^{2}}{(\theta-\tau)^{2}}
\end{aligned}
$$

By hypothesis, both $n$ and $r$ are powers of $q$ and so we deduce that $\theta-\tau$ is a power of the prime $p$. As $m_{\theta} m_{\tau}$ is an integer it now follows from (7.1) that $(\theta-\tau)^{2}$ divides $n^{2}$.

As $n$ and $r$ are both powers of $p$, it follows from the identity

$$
n-2-\delta=r c_{2}
$$

that $p$ divides $\delta+2$. Because $\theta$ and $\tau$ are zeros of the quadratic $x^{2}-$ $\delta x-(n-1)$, we find that $\delta=\theta+\tau$ and

$$
(\theta-\tau)^{2}=\delta^{2}-4+4 n,
$$

consequently $\theta-\tau$ divides $\delta^{2}-4$. If $\delta=-2$, then (7.2) yields that $4 n$ is a perfect square, $\theta-\tau$ is even and $p=2$.

Hence we assume that $\delta \neq-2$. Suppose that $p$ is odd. Then, as $\theta-\tau$ is a power of $p$ and as $\theta-\tau$ divides $\delta^{2}-4$, it follows that $\theta-\tau$ divides $\delta-2$ or $\delta+2$. Accordingly

$$
\theta-\tau \leq \delta+2=\theta+\tau+2
$$

This implies that $\tau$, the least eigenvalue of $X$, is at least -1 and therefore $X$ is disjoint union of cliques.

So $p=2$ if $\delta \neq-2$. Assume $n=2^{a}$ and $\theta-\tau=2^{s}$, where $s \leq a$. As $\theta \tau=1-n$, we find that

$$
(\theta+\tau)^{2}=(\theta-\tau)^{2}+4 \theta \tau=4\left(2^{2 s-2}-2^{a}+1\right)
$$

whence $2^{2 s-2}-2^{a}+1$ is a perfect square. Therefore there is an integer $\lambda$ such that

$$
2^{2 s-2}-2^{a}+1=\left(2^{s-1}-\lambda\right)^{2}=2^{2 s-2}-2^{s} \lambda+\lambda^{2} .
$$

Consequently

$$
\lambda 2^{s}-2^{a}=\lambda^{2}-1
$$


implying that $2^{s}$ divides $(\lambda-1)(\lambda+1)$. Hence $2^{s-1} \leq \lambda+1$, so $2^{s-1}-\lambda \leq$ 1 and (7.4) now yields that

$$
2^{2 s-2}-2^{a}+1=1 .
$$

Now (7.3) implies that $(\theta+\tau)^{2}=4$. As we have assumed $\delta \neq-2$, we must have that $\delta=2$.

The identity $n-2-\delta=r c_{2}$ now yields that $r$ divides 4 . If $r=4$ then

$$
c_{2}=\frac{n}{4}-1
$$

and so $c_{2}$ is odd, or $n=4$. As $r \leq n-1$, we cannot have $n=4$ and, by our feasibility Condition (F2) in Section 2, we know that $c_{2}$ is even if $n$ is even.

We have shown that, if $\delta \neq-2$ then $\delta=2$ and $r=2$. A two-fold cover of $K_{n}$ such that $\delta=2$ is one of a complementary pair with a cover with $\delta=-2$. Therefore $n$ is a perfect square in this case too. Q.E.D.

One consequence of this result is that, in odd characteristic, any pseudo-complement to a subspace is a subspace.

We derive another consequence of our work. Let us say that the association scheme determined by an equitable partition of $P G(d, q)$ has dimension $d$ over $G F(q)$. Any partition of a projective line is equitable, so there is little to say in that case.

Lemma 7.2. Let $X$ be antipodal distance-regular cover of a complete graph that is linear over $G F(q)$ with dimension two and has $\delta=-2$. Then $X=X(\Omega)$, where $\Omega$ is an oval.

Proof. Suppose $X$ is constructed from the equitable partition

$$
\left(\Omega_{1}, \Omega_{2}, \Omega_{3}\right) .
$$

Because $d=2$, we have $t=0$, so $\Omega_{3}$ is a point and $\left|\Omega_{1}\right|=q+1$. Further $r=q$ and $|X|=q^{3}$. As $\delta=-2$, we have $c_{2}=q$ and $a_{1}=q-2$. Now Theorem 5.4(d) yields that no line meets $\Omega_{1}$ in more than two points, and therefore $\Omega$ is an oval.

Q.E.D.

The Hermitian forms graph $\operatorname{Her}(2,3)$ has an antipodal distanceregular cover with diameter four and index three. (See $[4, \S 11.3 \mathrm{H}]$.) In analogy to our last result, Tilla Schade [24] has shown that there is a unique linear cover of this graph, and also given a new construction of it. Both these tasks are much more difficult than what we have just done. 


\section{$\S 8$. Codes}

Let $\mathcal{P}$ be a hyperplane in $P G(d+1, q)$, let $\omega$ be an equitable partition of it, with cells $\Omega_{1}, \ldots, \Omega_{s}$ and let $\omega^{*}$ be the induced partition on the hyperplanes of $\mathcal{P}$, with $\Omega^{*}{ }_{1}, \ldots, \Omega^{*}{ }_{s}$ as its cells. Let $\Omega$ denote $\Omega_{i}$ and let $M$ be a matrix whose columns are homogeneous coordinate vectors for the points in $\Omega$. We call the row space of $\Omega$ its code, and denote it by $C(\Omega)$. Each code word can be written in the form $x^{T} M$. As $x^{T}$ can be viewed as the homogeneous coordinate vector of a hyperplane, $H$ say, in $\mathcal{P}$, it follows that the weight of $x^{T} M$ is equal to

$$
|\Omega|-|H \cap \Omega| \text {. }
$$

All hyperplanes in the same cell of $\Omega^{*}$ give rise to words of the same weight, $w_{i}$ say. Hence the weight enumerator of $C(\Omega)$ is

$$
1+(q-1) \sum_{i=1}^{s}\left|\Omega_{i}^{*}\right| x^{w_{i}}
$$

The code words in $C(\Omega)$ form an $s$-class association scheme, where a pair of words $(u, v)$ is in the $i$-th class if $u-v$ has weight $w_{i}$. By a result of Delsarte [4, Thm. 2.10.13], its dual code is completely regular. The coset graph of the dual code is $X(\Omega)$.

We now consider the codes obtained from subsets $\Omega$ such that $X(\Omega)$ is an antipodal distance-regular cover of complete graph. Assume that $X(\Omega)$ has dimension $d$,over $G F(2)$, that its index $r$ is $2^{t+1}$ and that $d-t=2 e$. Then $X$ has parameters

$$
n=2^{2 e}, r=2^{t+1} ; \quad \theta=2^{e}-1, \tau=-2^{e}-1
$$

and the multiplicities of the eigenvalues of $X(\Omega)$ are $1,2^{2 e}-1$ and

$$
2^{e-1}\left(2^{t+1}-1\right)\left(2^{e}-1\right), \quad 2^{e-1}\left(2^{t+1}-1\right)\left(2^{e}+1\right) .
$$

Hence the weights and frequencies of the non-zero words in $C(\Omega)$ are as follows:

\begin{tabular}{c|c} 
weight & frequency \\
\hline $2^{2 e-1}-2^{e-1}$ & $2^{e-1}\left(2^{t+1}-1\right)\left(2^{e}-1\right)$ \\
$2^{2 e-1}$ & $2^{2 e}-1$ \\
$2^{2 e-1}+2^{e-1}$ & $2^{e-1}\left(2^{t+1}-1\right)\left(2^{e}+1\right)$ \\
\hline
\end{tabular}

Our code $C(\Omega)$ has odd length, if we add the binary complement of each code word and then add a parity check to each word, the weights and frequencies of the non-zero words in the resulting code are as follows: 


\begin{tabular}{c|c} 
weight & frequency \\
\hline $2^{2 e-1}-2^{e-1}$ & $2^{2 e}\left(2^{t+1}-1\right)$ \\
$2^{2 e-1}$ & $2^{2 e+1}-2$ \\
$2^{2 e-1}+2^{e-1}$ & $2^{2 e}\left(2^{t+1}-1\right)$ \\
$2^{2 e}$ & 1 \\
\hline
\end{tabular}

Denote this code by $K(\Omega)$. As $r<n$ we must have $t \leq 2 e-2$. If $t=2 e-2$ then $K(\Omega)$ has the same weight distribution as the Kerdock code of the same length. (See, e.g., [22, p. 456].) Since it follows from Brouwer and Tolhuizen [6] that there is no linear code with the same weight distribution as a Kerdock code, we deduce that a cover with $t=2 e-2$ cannot be linear.

The Preparata covers have parameter sets of the form $\left(2^{2 e}, 2^{2 e-1}, 2\right)$; the above argument shows that that these covers cannot be linear. But more is true: if $e>2$ then from [7] we know that the automorphism group of a Preparata cover is not vertex transitive.

\section{$\S$ 9. Questions}

A. As $\delta= \pm 2$ for a linear $\left(n, r, c_{2}\right)$-cover, it follows that $r$ divides $n$ and, as $n$ must be a power of two, $r \leq n / 2$. Our work in Section 8 shows that we cannot have $r$ equal to $n / 2$. Therefore $r \leq n / 4$. We ask:

(1) Is there a linear $\left(n, r, c_{2}\right)$-cover with $r^{2}>n$ ?

The smallest open case is when $n=64$. Here there are quotients of a Preparata cover with $r=16$, but we do not know if any are linear. The linear covers we do have are obtained as quotients of graphs $X(\Omega)$, where $\Omega$ is an oval in $P G(2, q)$ and $q$ is even; for these $r^{2} \leq n$.

B. A number of covers arise as the set of vertices at distance two from a vertex in a strongly regular graph. Examples are the 6-fold cover of $K_{7}$, and the covers obtained from generalised quadrangles with parameters $(q-1, q+1)$, which arise as second neighbourhoods in the point graphs of the symplectic generalised quadrangles. The strongly regular graphs such that each second neighbourhood is an antipodal distance-regular graph are determined in [14]. However there are examples of strongly regular graphs where just one second neighbourhood is a cover. This prompts the following:

(2) Are there covers which arise as second neighbourhoods of strongly regular graphs, with parameter sets different from those given in [14]?

The smallest open cases arise from covers with parameter sets

$$
(33,3,9),(36,4,8),(36,6,6) \text {. }
$$


If $X$ is a $\left(n, r, c_{2}\right)$-cover and occurs as the second neighbourhood of a strongly regular graph $Y$, then the parameters of $Y$ are determined as follows. Set $\gamma$ equal to $c_{2}\left(c_{2}-1\right) /\left(n-c_{2}\right)$. Then $Y$ has $(r+1) n+c_{2}+\gamma$ vertices, valency $n-1+c_{2}+\gamma$, two adjacent vertices have exactly $a_{1}+\gamma$ common neighbours and two vertices at distance two have exactly $c_{2}+\gamma$ common neighbours. It is clearly necessary that $\gamma$ be an integer; it is also necessary to check that the output of the formulas for the multiplicities of the eigenvalues of $Y$ are integers. In the three cases in (9.1), I do not even know that strongly regular graphs with the required parameters exist, let alone have the required second neighbourhoods.

Remark. Don de Caen (private communication) has shown that a $(33,3,9)$-cover cannot occur as the second neighbourhood of a strongly regular graph.

C. We have seen that covers with $\delta$ in $\{-2,0,2\}$ are special, and we have large classes of examples with $\delta$ equal to 0 or -2 . The supply with $\delta=2$ is much more limited, the only examples arise from generalised quadrangles with parameters $(q+1, q-1)$ and then $n$ is a power of two. Thus we ask:

(3) Is there a cover with $\delta=2$ where $n$ is divisible by an odd prime?

All the covers we know with $\delta=-2$ have a prime power number of vertices; hence we also ask:

(4) Is there a cover of $K_{n}$ such that $\delta=-2$ and $n$ is not a prime power?

There are a number of parameter sets such that $\delta= \pm 2$ and $n=36$ for which no covers have been found, but all known feasibility conditions are satisfied. In particular, the second and third parameter sets in (9.1) have $\delta=2$ and -2 respectively.

Finally we list the parameter sets with $n \leq 25$ where we do not know if a cover exists.

\begin{tabular}{c|c|c|c|c} 
& $n$ & $r$ & $c_{2}$ & $\delta$ \\
\hline $\mathbf{1 .}$ & 13 & 11 & 1 & 0 \\
$\mathbf{2 .}$ & 16 & 7 & 2 & 0 \\
$\mathbf{3 .}$ & 19 & 17 & 1 & 0 \\
$\mathbf{4 .}$ & 21 & 4 & 5 & -1 \\
$\mathbf{5 .}$ & 21 & 10 & 2 & -1 \\
$\mathbf{6 .}$ & 22 & 6 & 4 & -4 \\
$\mathbf{7 .}$ & 25 & 3 & 7 & 2 \\
$\mathbf{8 .}$ & 25 & 7 & 3 & 2 \\
$\mathbf{9 .}$ & 25 & 21 & 1 & 2 \\
\hline
\end{tabular}


Of course, the existence of covers 1 . or 3 . would imply the existence of a projective plane of order 12 or 18 respectively. These entries are included only for completeness. The cover of $K_{22}$ is interesting in that it would be triangle-free.

\section{References}

[1] N. L. Biggs, Distance-regular graphs with diameter three, Ann. Discrete Math., 15 (1982), 69-80.

[2] R. C. Bose and W. S. Connor, Combinatorial properties of group divisible incomplete block designs, Ann. Math. Stat., 32 (1952), 367-383.

[ 3 ] W. G. Bridges and R. A. Mena, On the rational spectra of graphs with abelian Singer groups, Linear Algebra Appl., 46 (1982), 51-60.

[4] A. E. Brouwer, A. M. Cohen and A. Neumaier, "Distance-Regular Graphs", Springer, Berlin, 1989.

[5] A. E. Brouwer, C. D. Godsil and H. A. Wilbrink, Isomorphism between antipodal distance-regular graphs with diameter three, unpublished manuscript.

[6 ] A. E. Brouwer and L. M. G. M. Tolhuizen, A sharpening of the Johnson bound for binary linear codes and the non-existence of linear codes with Preparata parameters, Des. Codes Cryptogr., 3 (1993), 95-98.

[7] D. de Caen, R. Mathon and G. E. Moorhouse, A family of antipodal distance-regular graphs related to the classical Preparata codes, J. Algebraic Combin., to appear.

[8] A. R. Calderbank and W. M. Kantor, The geometry of two-weight codes, Bull. London Math. Soc., 18 (1986), 97-122.

[9] P. J. Cameron, Covers of graphs and EGQ's, Discrete Math. 97 (1991) 83-92.

[10] P. J. Cameron and J. H. van Lint, "Designs, Graphs, Codes and their Links", London Math. Soc. Student Texts 22, Cambridge U. P., Cambridge, 1991.

[11] P. Delsarte, An algebraic approach to the association schemes of coding theory, Philips Research Reports Supplements 1973, No. 10.

[12] A. D. Gardiner, Antipodal covering graphs, J. Combin. Theory Ser. B, 16 (1974) 255-273.

[13] A. D. Gardiner, Imprimitive distance-regular graphs and projective planes, J. Combin. Theory Ser. B, 16 (1974) 274-281.

[14] A. D. Gardiner, C. D. Godsil, A. D. Hensel and G. F. Royle, Second neighbourhoods of strongly regular graphs, Discrete Math. 103 (1992) 161-170.

[15] C. D. Godsil, "Algebraic Combinatorics", Chapman and Hall, New York, 1993.

[16] C. D. Godsil, Krein covers of complete graphs, Australas. J. Combin., 6 (1992) 245-255. 
[17] C. D. Godsil, Geometric distance-regular covers, New Zealand J. Math., 22 (1993) 31-38.

[18] C. D. Godsil and A. D. Hensel, Distance regular covers of the complete graph, J. Combin. Theory Ser. B, 56 (1992), 205-238.

[19] W. M. Kantor, Projective planes of order $q$ whose collineation groups have order $q^{2}$, J. Algebraic Combin., 3 (1994), 405-425.

[20] P. W. H. Lemmens and J. J. Seidel, Equiangular lines, J. Algebra, 24 (1973), 494-512.

[21] H. Lüneburg, "Translation Planes", Springer, Berlin, 1980.

[22] F. J. MacWilliams and N. J. A. Sloane, "The Theory of Error-Correcting Codes", North-Holland, Amsterdam, 1977.

[23] S. Payne and J. A. Thas, "Finite Generalised Quadrangles", Pitman, New York, 1985.

[24] T. Schade, Antipodal Distance-Regular Graphs, Ph. D. Thesis, Universität Gießen, 1994.

[25] J. J. Seidel, "Geometry and Combinatorics, Selected works of J. J. Seidel", Eds. D. G. Corneil and R. Mathon, Academic Press, San Diego, 1991.

Combinatorics and Optimization

University of Waterloo

Waterloo, Ontario

Canada N2L 3G1 